\title{
Aktionäre durch Online Communities einbinden
}

Börsennotierte Unternehmen erkennen vermehrt, wie attraktiv Online Communities für ihre Finanzkommunikation sind. Vor einem Einsatz sollten Unternehmen die verfügbaren Optionen aber sorgfältig prüfen. Forschungsergebnisse geben Aufschluss, wie eine erfolgreiche Einbindung von Aktionären über Online-Plattformen gelingen kann.

Christian Pieter Hoffmann 
Digitale Medien sind heute ein alltäglicher Bestandteil der Finanzkommunikation. Ihr Einsatz gehört zu den guten Geschäftspraktiken der Investor Relations (IR). Kein DAX, M-, S- oder TecDAX-Unternehmen kann auf eine professionell gepflegte IR-Webseite verzichten. Diverse Rankings analysieren und prämieren jährlich die Funktionalität und Nutzerfreundlichkeit von IR-Webseiten.

Die Popularität digitaler Medien in der Finanzkommunikation ist wenig verwunderlich, denn das Internet bietet ihr zahlreiche Vorteile:

- fokussierte Ansprache der interessierten Zielgruppe,

- simultane Abdeckung diverser Informationsbedürfnisse,

- hohe Geschwindigkeit der Kommunikation,

- Angebot einer großen Informationsbreite und -tiefe und

- Multimedialität (Schriftdokumente, Präsentationen, Ton- und Videoaufnahmen).

Nicht zuletzt stellt eine gut gepflegte IR-Webseite eine Entlastung des IRTeams dar, denn zahlreiche Informationswünsche der Investoren, Privataktionäre, Analysten oder Journalisten können hier „abgefangen“ werden. Eine persönliche Interaktion wird erst nötig, wenn die Suchenden im Netz nicht fündig geworden sind.

\section{Neue Medien in der Finanzkommunikation}

Der aktive Einsatz neuer Medien in der Finanzkommunikation ist jedoch durchaus keine Selbstverständlichkeit. Unter den diversen Kommunikationsfunktionen des Unternehmens sind die Investor Relations zweifellos die zurückhaltendste, wenn es um das Aufgreifen und den Einsatz sozialer Medien geht. Blogs, soziale Netzwerke oder Twitter finden nur zögerlich ihren

\section{„Blogs, soziale Netzwerke oder Twitter finden nur zögerlich ihren Weg in die IR-Praxis."}

Weg in die IR-Praxis. Zögerlich - aber immerhin doch spürbar (vgl. Fieseler et al. 2010). Aufgrund der hohen Geschwindigkeit und überschaubaren Länge der hier verbreiteten Mitteilungen hat sich etwa Twitter als populäres Kommunikationsmedium im Kapitalmarkt etabliert. Auch das Teilen von Inhalten auf Content-Plattformen wie etwa Slideshare hat in Ergänzung der IR-Webseiten eine gewisse Verbreitung gefunden. Weniger populär ist es, ausführliche Informationen auf Blogs zu veröffentlichen. Und geradezu stiefmütterlich werden bisher soziale Netzwerke wie Facebook, Xing oder LinkedIn behandelt.

Dabei entfalten sich gerade in sozialen Netzwerken jene Dynamiken, welche die neuen, sogenannten „Mitmach-Medien“ auszeichnen. Individuelle Nutzer unterhalten hier Profile, um sich untereinander zu vernetzen und in diesen Netzwerken Informationen zu teilen. Aufgrund gemeinsamer Interessen bilden sich Gemeinschaften - sei dies bottom-up, indem einzelne

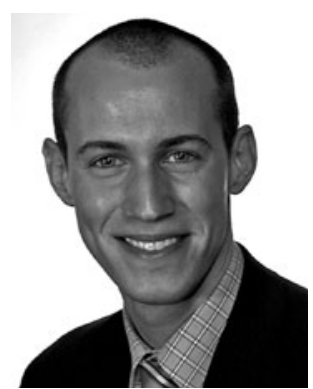

Prof. Dr. Christian Pieter Hoffmann ist Assistenzprofessor am Institut für Medien- und Kommunikationsmanagement der Universität St. Gallen (CH), Tel.: +41 (0)71 224-3024,

E-Mail: christian.hoffmann@unisg.ch 
Nutzer Anschluss untereinander suchen und finden, oder sei es top-down, indem Organisationen interessierte Nutzer um sich scharen und so in den unmittelbaren Austausch mit relevanten Zielgruppen eintauchen.

„Mitmach-Medien“ sind die neuen Online-Plattformen vor allem deshalb, weil sie ihren Nutzern das Veröffentlichen von Daten - Mitteilungen, Bildern, Empfehlungen, Videos, Links oder Präsentationen - so leicht wie möglich machen. „Mitmach-Medien“ sind sie aber auch, weil sich die Nutzer auf dieser Grundlage in lebendige Austausch-Prozesse einbringen, sie schalten sich ein, sie teilen ihre Meinung mit (vgl. Kane et al. 2013). Soziale Medien zeichnen sich durch eine Beteiligungskultur aus, die keineswegs nur politischer oder auch stark formalisierter Natur sein muss. Beteiligung kann schon die autonome Selektion relevanter Informationen im Austausch mit diversen Kontakten sein, die Äußerung von Unzufriedenheit oder die Abgabe einer Empfehlung.

\section{Die Attraktivität von Online Communities}

Betrachtet man einige aktuelle Herausforderungen der Finanzkommunikation, wird schnell deutlich, warum „Mitmach-Medien “ in Form von Online-Gemeinschaften auch für die IR ein interessantes Betätigungsfeld darstellen können.

Aufmerksamkeitsökonomie: In der tosenden Stimmenvielfalt, die nicht zuletzt durch die immer zahlreicheren Informationsplattformen im Netz befördert wird, ist es für Unternehmen immer schwieriger, Aufmerksamkeit bei ihren Zielgruppen zu gewinnen. Wie kann es Unternehmen also gelingen, zu ihren relevanten Zielgruppen durchzudringen? Als etablierter, respektierter Bestandteil einer Gemeinschaft positioniert sich ein Kommunikator an den Schnittstellen der Kommunikationsflüsse zwischen den Mitgliedern. Es fällt

\section{Zusammenfassung}

- Online Communities können der effizienten Ansprache, Information, Aktivierung und Einbindung von Aktionären dienen.

- Heute werden diverse, unterschiedlich proprietäre Lösungen für Shareholder-Communities angeboten.

- Um eine Online Community zweckmäßig für die Finanzkommunikation einsetzen zu können, sollten Unternehmen die spezifische Dynamik von Online Communities kennen und verstehen. ihm darum leichter, gezielt eigene Inhalte einzusteuern und so tatsächlich Wahrnehmung zu erfahren. Soziale Netzwerkanalysen zeigen, dass die Netzwerkzentralität eines Akteurs wesentlich für das Erreichen eines breiten Publikums ist - und dies direkt, vermittelt durch möglichst wenige Intermediäre.

Aktivierung und Mobilisierung: Nicht erst seit den berüchtigten Betrugsfällen rund um Großkonzerne wie Enron oder Parmalat ist die Corporate Governance ein, wenn nicht das zentrale Thema der Kapitalmarktkommunikation. Mit zunehmender öffentlicher Aufmerksamkeit für das Fehlverhalten einzelner Firmen steigt zugleich der politische Druck

\section{„Die Funktion von Online Communities im spezifischen Anwendungskontext der Finanzkommunikation ist bislang noch wenig erforscht."}

auf Kapitalgesellschaften, eine Kontrolle und Aufsicht durch die Aktionäre zu fördern und zu unterstützen. Paradoxerweise verliert die Teilnahme an Hauptversammlungen jedoch gleichzeitig beständig an Popularität. Zunehmend müssen sich Unternehmen mächtig ins Zeug legen, um eine nennenswerte Zahl interessierter Investoren zu einer Teilnahme zu bewegen - und so mögliche Störmanöver durch Minderheitsaktionäre zu erschweren. Wenn das Engagement in Online Communities tatsächlich einen involvierenden und mobilisierenden Effekt ausübt, sollte dies von hoher Attraktivität für die betroffenen Unternehmen sein (vgl. Bogan 2008).

Effizienz: Die Finanzkommunikation ist bereits die am stärksten regulierte Kommunikationsdisziplin des Unternehmens, und der Umfang der Publikations- und Kommunikationspflichten nimmt in der Tendenz immer weiter zu. Wie können Unternehmen sicherstellen, dass die richtigen Informationen die richtigen Zielgruppen fristgerecht erreichen? In Online Communities besteht ein direkter Draht zwischen den Mitgliedern. Sie stellen eine Art geschützten Raum dar, in dem die obligatorischen, und darüber hinaus weitere, freiwillige Informationen direkt an die Aktionäre übermittelt werden können. Diese können online die relevanten Daten ablegen und organisieren. Selbstverständlich wird dadurch die gleichberechtigte Information der Öffentlichkeit nicht substituiert. Die Erfüllung der Publikationspflichten - und Vermeidung allfälliger Sanktionen infolge möglicher Fehltritte wird dennoch erleichtert. 
Intelligence: Online Communities sind ein praktisches Instrument zur Verbreitung von Informationen, vor allem für gut positionierte oder vernetzte Akteure. Als „Mitmach-Medium " sind sie aber nicht auf eine Ein-Wege-Kommunikation getrimmt. Im Gegenteil: Die Konversation unter den Mitgliedern bildet das Kapital der Gemeinschaft. Unternehmen müssen, ja sollten daher auch in Online Communities nicht nur auf Senden eingestellt sein. Mindestens so wertvoll ist das $\mathrm{Zu}$ hören. In einer Online-Gemeinschaft hat ein Unternehmen Auge und Ohr direkt an der Konversation seiner Zielgruppe. Durch ein regelmäßiges Monitoring, oder auch schlicht durch das gezielte Einholen eines Feedbacks in Form einer Frage oder Abstimmung, lassen sich frühzeitig Stimmungen - ob positive oder negative - erkennen. Kommunikative Reaktionen können eingeleitet werden, lange bevor sich ein Frustpegel aufbaut, der sich schließlich im Rahmen der Hauptversammlung lautstark entlädt.

\section{Erste Gehversuche}

Immer mehr Anbieter erkennen die Attraktivität von Online Communities auch für die Finanzkommunikation und bemühen sich, entsprechende Applikationen zu entwickeln. Heutige Ansätze unterscheiden sich in der Breite unterstützter Funktionalitäten, insbesondere von Möglichkeiten der Interaktion auf der Plattform, sowie in der Offenheit des Angebots. Vor allem basierend auf letzterem Kriterium lassen sich drei Lösungsansätze definieren.

Unternehmen können zunächst autonome Angebote für ihre Finanzkommunikation nutzen. Hierzu zählen Communities, in denen Unternehmen zwar präsent sein können, etwa durch eigene Profile, Foren oder Werbung, die jedoch unabhängig von diesen angeboten werden und sich unmittelbar an die Aktionäre wenden. Beispiele sind soziale Netzwerke für Aktionäre, in denen diese sich Profile anlegen, untereinander vernetzen und zu diversen Themen rund um die Kapitalmärkte austauschen. Eine gewisse Bekanntheit hat etwa die amerikanische Plattform Stocktwits erreicht, auf der Twitter-ähnliche Nachrichten zu Investitionschancen verbreitet werden.

Ein anderer Ansatz ist, mehr oder minder maßgeschneiderte Lösungen für den Unterhalt einer Shareholder Online Community zu nutzen, die einige Dienstleister, häufig Startups, Unternehmen anbieten. Der Vorteil solcher intermediären Angebote ist, dass zwar der Austausch zwischen dem Unternehmen und seinen Investoren im Vordergrund steht, jedoch gleichzeitig mehrere Unternehmen jeweils mit einem geschützten Bereich auf der Plattform präsent sein können. Dies macht die Gesamtplattform für die Aktionäre attraktiver.

Unternehmen, die nicht auf den Plattformen eines Drittanbieters eine Präsenz unterhalten wollen, können natürlich auch eine eigenständige Lösung entwickeln und anbieten. In den USA unterhält etwa das ohnehin für seine Social-Media-Affinität bekannte Unternehmen Comcast eine eigene Shareholder-Community-Plattform. Im deutschsprachigen Raum gehörte Daimler zu den ersten Anbietern einer Online-Plattform für das eigene Aktionariat. Vorteile eines solchen proprietären Angebots sind dessen Autonomie und die unmittelbare Kontrolle des Unternehmens über das Angebot. Nachteilig wirken sich jedoch die Entwicklungs- und Unterhaltskosten aus, vor allem wenn die Aktionäre wiederholt zu einem Engagement auf der Plattform animiert werden müssen.

Innerhalb der beschriebenen drei Ansätze gibt es eine Vielfalt an Funktionalitäten, wie etwa die Möglichkeit zur Einrichtung und Gestaltung eines Nutzerprofils, Applikationen der Kommunikation zwischen den Community-Mitgliedern und dem Unternehmen sowie unter den Mitgliedern, synchrone und asynchrone Kommunikationsmittel, Abstimmungs- und Umfragemodule und vieles mehr. Vor allem im Falle intermediärer und proprietärer Angebote ist auch die Anbindung an elektronische Abstimmungen im Rahmen von Hauptversammlungen zumindest technisch ohne Weiteres möglich und umsetzbar.

\section{Kernthesen}

- Neue Medien sind „Mitmach-Medien“, sie bewirken die Aktivierung ihrer Nutzer.

- Online Communities können der Ansprache, Information und Einbindung von Aktionären dienen.

- Es bestehen diverse Angebote für Aktionärs-Communities, die eine unterschiedliche Kontrolle der Unternehmen zulassen.

- Online Communities zeichnen sich durch unterschiedliche Nutzungszwecke und -intensitäten ihrer Mitglieder aus.

- Aktives Engagement in Online Communities fördert die Identifikation und die Bindung der Zielgruppen an das Unternehmen. 


\section{Erfolgsfaktoren von Online Communities}

Wenn nun also Online Communities für den Einsatz in der Finanzkommunikation geeignet und verfügbar sind, was sollten interessierte Unternehmen für eine erfolgreiche Nutzung solcher Applikationen beachten? Die Funktion von Online Communities im spezifischen Anwendungskontext der Finanzkommunikation ist bislang noch wenig erforscht. Eine gewisse Aufmerksamkeit haben solche Communities jedoch zum Zwecke der Kundenkommunikation gefunden - etwa im Fall der Open Innovation und des Crowdsourcings (vgl. Sashi 2012). Selbstverständlich existieren zahlreiche Online Communities auch außerhalb des unternehmerischen Kontextes, etwa jene, die kulturellen oder Bildungszwecken gewidmet sind. Aus Untersuchungen solcher Gemeinschaften lassen sich vier verallgemeinerbare Erkenntnisse für das Community Management ableiten.

Erstens, Nutzungszwecke unterscheiden sich und sind wandelbar. Mitglieder finden meist aufgrund eines funktionalen Bedürfnisses Zugang zu einer Community. Schon diese funktionalen Nutzungsmotive können sich jedoch unterscheiden, etwa wenn eine Information gesucht wird, ein Vorschlag diskutiert oder eine Person oder Gruppe adressiert werden soll. Über funktionale Bedürfnisse hinaus gibt es jedoch auch soziale Nutzungsmotive. Je länger Mitglieder eine Online Community nutzen, desto wichtiger wird ihnen der Austausch untereinander,

\section{Handlungsempfehlungen}

- Online Communities müssen für die Aktionäre mindestens so attraktiv sein wie für das Unternehmen. Vermeiden Sie Insellösungen. Sie sind für die Nutzer wenig effizient.

- Online Communities sind sozial. Darum sollten Sie die Interaktion der Aktionäre untereinander zulassen und unterstützen.

- Online Communities basieren auf Identifikation. Eine Personalisierung der Nutzeransprache fördert die Einbindung und regt Engagement an.

- Online Communities weisen unterschiedliche Beteiligungsgrade auf. Lassen Sie sich nicht beunruhigen, wenn sich ein Großteil der Nutzer überwiegend passiv verhält.

- Besonders aktive Nutzer sorgen für attraktive und lebendige Communities. Identifizieren und fördern Sie diese Nutzer. das rein funktionale Nutzungsmotiv rückt in den Hintergrund (vgl. Nov et al. 2008). Unternehmen ist daher zu empfehlen, den direkten Austausch unter den Mitgliedern zu ermöglichen.

Zweitens, Nutzertypen unterscheiden sich. Eine etablierte Typologie von Community-Mitgliedern (vgl. Ewing 2008) unterscheidet „Newbies“, also Neumitglieder, von „Regulars“, regelmäßigen Besuchern, „Elders“, besonders erfahrenen Mitgliedern, und "Legacies", vormalige Elders, die sich zunehmend von der Plattform zurückziehen. „Lurkers“ werden jene Mitglieder genannt, die vor allem passiv Inhalte konsumieren, ohne selbst aktiv zu werden. Und „Trolls“ sind schließlich jene Störenfriede, die durch provokative Mitteilungen Dissens erzeugen wollen. Erfahrungsgemäß sind Elders besonders wichtig für die inhaltliche Ausrichtung, Tonalität und Lebendigkeit einer Plattform. Solche Mitglieder sollten Unternehmen daher pflegen und möglicherweise auch belohnen - sei es durch eine herausgehobene Präsentation auf der Plattform, durch eine Kennzeichnung in Form entsprechender Rollentitel oder auch durch materielle Anreize, wie etwa eine besondere Betreuung oder Verpflegung im Rahmen der Hauptversammlung.

Drittens, Nutzungsintensitäten unterscheiden sich. Das Engagement der Mitglieder in einer Online Community folgt in der Regel einer Potenzverteilung. Sehr wenige Mitglieder sind sehr aktiv, einige Mitglieder weisen eine gewisse Aktivität auf, und die Masse der Mitglieder ist wenig bis gar nicht aktiv. Diese Ungleichverteilung wirkt häufig verstörend auf unerfahrene Anbieter. Schnell wird eine Community für gescheitert erklärt, weil sich nur wenige Mitglieder tatsächlich aktiv einbringen. Dabei zeigt die Forschung: Die besonders aktiven wenigen dienen der Organisation und Belebung der Plattform, die mäßig Aktiven tragen jene Inhalte bei, die die Community lebendig halten, und die Masse der Mitglieder manifestiert durch ihre Inaktivität nicht zuletzt Zustimmung oder Zufriedenheit. Aus dem Kontext der Open Innovation ist bekannt, dass gute Ideen auch dann entstehen, wenn sich die meisten Teilnehmenden gar nicht aktiv einbringen.

Viertens, Nutzung verbindet. Die bewusste Entscheidung zur Teilnahme an einer Online Community ist ein Akt der Identifikation. Diese Identifikation wird weiter gestärkt durch den Verlauf des Engagements auf der Plattform. Community-Mitglieder entwickeln ein Zugehörigkeitsgefühl, eine Art "Citizenship“ (Yen et al. 2011). Dieser „Buy-in“ eines Community-Mitglieds stellt gerade für Unternehmen eine wertvolle Ressource dar. Durch die Personalisierung der Begrüßung, Einführung, Interaktion und auch der Selbstdarstellung der Mitglieder kann dieser Effekt gefördert werden. 


\section{Fazit}

Die Finanzkommunikation ist zweifellos eine spezifische Domäne, geprägt durch eine ganz eigene Kultur und funktionalistische Logik. Kapitalmarktteilnehmer reagieren gelegentlich geradezu allergisch auf alles, was allzu weich und spielerisch daherkommt. Es gibt immer noch weitverbreitete Vorbehalte gegen soziale Medien, die diesen Habitus dokumentieren. Sicher lassen sich nicht alle Forschungsergebnisse zur Funktion von Online Communities auf die Finanzkommunikation übertragen. Die bisherigen Erfahrungen deuten jedoch darauf hin, dass ein überzeugender Business Case für den Einsatz von Online Communities in der Finanzkommunikation dargestellt werden kann. Interessierte Unternehmen sollten die verfügbaren Optionen abwägen. Und sie sollten frühzeitig Informationen zur spezifischen Dynamik von Online Communities einholen, einerseits um die richtigen Anreize für deren Mitglieder zu setzen, andererseits um unrealistische Erwartungen und Enttäuschungen zu vermeiden. Auf dieser Grundlage können die Information, Aktivierung und Einbindung der Zielgruppen durch Community-Angebote gelingen.

\section{Literatur}

Bogan, V.: Stock market participation and the Internet, in: Journal of Financial and Quantitative Analysis, 43. Jg. (2008), Heft 1, S. $191-211$.

Ewing, T.: Forum - Participation cycles and emergent cultures in an online community, in: International Journal of Market Research, 50. Jg. (2008), Heft 5, S. 575 - 590.

Fieseler, C./Hoffmann, C./Meckel, M.: IR 2.0: Soziale Medien in der Kapitalmarktkommunikation, Hamburg 2010.

Kane, G. C./Alavi, M./Labianca, G./Borgatti, S. P.: What's Different about Social Media Networks? A Framework and Research Agenda, in: MIS Quarterly (im Druck).

Nov, O./Naaman, M./Ye, C.: Analysis of Participation in an Online Photo-Sharing Community: A Multidimensional Perspective, in: Journal of the American Society for Information Science and Technology, 61. Jg. (2009), Heft 3, S. 555 - 566.

Sashi, C. M.: Customer engagement, buyer-seller relationships, and social media, in: Management Decision, 50. Jg. (2012), Heft 2, S. $253-272$.

Yen, H. R./Hsu, S. H./Huang, C.: Good Soldiers on the Web: Understanding the Drivers of Participation in Online Communities of Consumption, in: International Journal of Electronic Commerce, 15. Jg. (2011), Heft 4, S. 89 - 120.

SISP] Zusätzlicher Verlagsservice für Abonnenten von „Springer für Professionals | Finance \& Controlling“

Zum Thema "Investor Relations" „Social Media“

\section{Medium}

$\square$ Online-Artikel (2)

$\square$ Zeitschriftenartikel (5)

$\square$ Buchkapitel (65)

\section{Sprache}

$\square$ Deutsch (59)

$\square$ Englisch (13)
Von der Verlagsredaktion empfohlen

Barrantes, E./Stärz, H.: Gläubigerorientierte Finanzkommunikation in börsennotierten Unternehmen. Forschungsstand und neue empirische Befunde, in: Hasler, P. T./Launer, M. A./Wilhelm, M. K. (Hrsg.): Praxishandbuch Dept Relation, Wiesbaden 2013, S. 29 - 47, www.springerprofessional.de/4415216

Clement, R./Schreiber, D.: Soziale Netzwerke und Social Media-Technologien, in: Clement, R./Schreiber, D.: Internet-Ökonomie, 2. Aufl., Berlin Heidelberg 2013, S. 395 - 461, www.springerprofessional.de/4522878 